\title{
Progress towards an interdisciplinary science of plant phenology: building predictions across space, time and species diversity
}

Author for correspondence: Elizabeth M. Wolkovich Tel: +1 6043765094 Email: lizzie@biodiversity.ubc.ca

Received: 31 July 2013 Accepted: 7 October 2013

\author{
Elizabeth M. Wolkovich ${ }^{1}$, Benjamin I. Cook ${ }^{2,3}$ and T. Jonathan Davies ${ }^{4}$ \\ ${ }^{1}$ Biodiversity Research Centre, University of British Columbia, Vancouver, BC V6T 1Z4, Canada; ${ }^{2}$ NASA Goddard Institute for Space \\ Studies, New York, NY 10025, USA; ${ }^{3}$ Ocean and Climate Physics, Lamont-Doherty Earth Observatory, Palisades, NY 10964, USA; \\ ${ }^{4}$ Department of Biology, McGill University, Montreal, QC H3A 1B1, Canada
}

New Phytologist (2014) 201: 1156-1162 doi: $10.1111 / \mathrm{nph} .12599$

Key words: climate change, cues, investment, local adaptation, phenology, plant ecology, plasticity, risk.

\section{Summary}

Climate change has brought renewed interest in the study of plant phenology - the timing of life history events. Data on shifting phenologies with warming have accumulated rapidly, yet research has been comparatively slow to explain the diversity of phenological responses observed across latitudes, growing seasons and species. Here, we outline recent efforts to synthesize perspectives on plant phenology across the fields of ecology, climate science and evolution. We highlight three major axes that vary among these disciplines: relative focus on abiotic versus biotic drivers of phenology, on plastic versus genetic drivers of intraspecific variation, and on cross-species versus autecological approaches. Recent interdisciplinary efforts, building on data covering diverse species and climate space, have found a greater role of temperature in controlling phenology at higher latitudes and for early-flowering species in temperate systems. These efforts have also made progress in understanding the tremendous diversity of responses across species by incorporating evolutionary relatedness, and linking phenological flexibility to invasions and plant performance. Future research with a focus on data collection in areas outside the temperate mid-latitudes and across species' ranges, alongside better integration of how risk and investment shape plant phenology, offers promise for further progress.

\section{Introduction}

Plant phenology - the timing of recurring life history events such as leafing and flowering - is an inherently interdisciplinary field of research with a history extending back hundreds of years. In plant ecology, phenology represents a major temporal component of ecosystem functioning, with important connections to carbon sequestration, food webs, and competition and coexistence (Gotelli \& Graves, 1996). It is also a major component of biometeorology, with many of the best estimates of phenological responses to temperature coming from European weather station records dating back hundreds of years (Cleland et al., 2007). More recently, phenology has been a focus in plant molecular ecology research, where studies of underlying physiological and genetic structures that control flowering have provided evolutionary insights into how local adaptation shapes intraspecific variation (Wilczek et al., 2010).
These various disciplines have tended to be largely independent. Ecology has traditionally worked on local-scale dynamics with a focus on how biotic interactions - especially with pollinators, seed dispersers and predators - may shape phenology (Mosquin, 1971; van Schaik et al., 1993). By contrast, biometeorology, with its prescribed aims to link biology and climate, has focused almost entirely on abiotic drivers. Thus, studies have often used a restricted number of phenologically plastic woody species, frequently planted as clones to minimize genetic variation (Schwartz et al., 2006; Cook et al., 2012a). Molecular studies have also been taxonomically restricted, but have focused more on genetic variation - with most studies examining clinal variation in phenology (Howe et al., 2003; Wilczek et al., 2010). More recently, phylogenetic approaches from evolutionary biology have also been applied to phenology integrating macroevolutionary time-scales and a more diverse species perspective, but again emphasizing the genetic basis of phenology (Davis et al., 2010). 
Table 1 Differences across the three major disciplines in plant phenology in their focus on what drives variation in plant phenology, how phenotypic plasticity versus genotypic local adaptation controls intraspecific variation, and the breadth of species generally studied

\begin{tabular}{llllll}
\hline Discipline & Major driver & Intraspecific variation & Species diversity & Temporal scale & Spatial scale \\
\hline Biometeorology & Abiotic & Plastic & Low & Large & Large \\
Ecology & Biotic $^{1}$ & Plastic and genetic & High & Short $^{2}$ & Local \\
Evolutionary biology & Abiotic $^{3}$ & Genetic $^{4}$ & Low $^{5}$ & Large $^{2}$ & Varies from local to the size of species' ranges \\
\hline
\end{tabular}

These varying foci lead then to varying dominant temporal and spatial scales across the disciplines. It should be noted that some fields of study cut across these disciplines. In particular, physiological studies of phenology are used in all three disciplines noted here and have been critical to the advancement of phenological research. An extended version of this table with relevant references is given the Supporting Information Table S1.

${ }^{1}$ Long focus on biotic drivers of selection, but recent work considers how climate and climate change effect phenology; for example, Inouye (2008).

${ }^{2}$ Generally studies examine dynamics over days to years; recent work integrates over decades; for example, Fitter \& Fitter (2002) and Inouye (2008).

${ }^{3}$ Much focus on clinal variation driven by climate; some studies also consider biotic interactions; see Table S1 for references.

${ }^{4}$ Much focus on heritable differences in phenology; however, research also highlights plastic responses; see Howe et al. (2003) for an overview.

${ }^{5}$ Molecular studies tend to focus on a handful of model laboratory and crop species; however, phylogenetic approaches focus on greater species diversity; see Kochmer \& Handel (1986), for example.

As climate change has brought renewed interest in phenology, there have been increasing efforts to integrate ideas and perspectives across disciplines, with the goal of building an improved framework for predicting plant phenology across species, time and space (Cleland et al., 2007; Pau et al., 2011). Here, we review recent work in plant phenology aimed at integrating across the fields of climate science, ecology and evolutionary biology. Specifically, we review how far research has progressed towards its goal of building a predictive framework, and consider what areas of future research appear crucial for robust predictions of plant phenological responses to climate change.

\section{Interdisciplinary perspectives on phenology}

We posit that phenological research across climate science, ecology, and evolutionary biology varies along three major axes: the relative focus on abiotic versus biotic forces driving phenology, the breadth of species studied, and the emphasis on plastic versus genetic responses leading to variation in phenology. Differences in the positioning along these axes allow each field to offer understanding at a unique temporal-spatial scale (Table 1). One major benefit then - and challenge - of an interdisciplinary approach to plant phenological research is integrating across these varying spatial and temporal scales. We review recent interdisciplinary advances below, highlighting conceptual efforts to integrate abiotic and biotic perspectives and empirical work across a diversity of species.

\section{Abiotic versus biotic drivers}

Predicting plant phenological responses requires understanding of when abiotic versus biotic forces drive selection on plant phenology (Pau et al., 2011). Much of the work in this area is grounded explicitly or implicitly in the concept of the temporal niche (Gotelli \& Graves, 1996) - that time is a primary axis by which plant species can partition resources, from nutrients to pollinators. Both abiotic and biotic forces may ultimately shape selection on plant phenology (Box 1). However, we expect that most species - even those with phenologies shaped almost entirely by biotic forces - proximately trigger phenology through abiotic cues. For example, selection on flowering time for some species may be driven to match the timing of specialist pollinators, but physiologically such species often use environmental cues such as temperature and photoperiod to cue flowering. Thus, we stress that our discussion in this section refers to ultimate abiotic and biotic drivers, and only to proximate abiotic triggers when referring to cues.

The relative balance of how selection on phenology is shaped by abiotic versus biotic drivers should depend both on the regional climate regime and on the position of a species' phenology within the growing season (Box 1). Regional climate sets the dominant controller of the growing season and thus should also define which climatic factor drives phenology for many species. In mesic temperate biomes, where precipitation is regular throughout the year and, thus, water rarely limiting, temperature is expected to be a major driver of phenology, especially at the start and end of the growing season when it is most variable; in mid-season though, when climate variability is low and competition between plants for soil, light and pollinator resources is high, biotic forces should dominate to shape selection (Box 1).

Outside of temperate mesic environments, many additional climatic factors control growing season length and periods when abiotic versus biotic drivers dominate. Moisture appears to be a critical driver of phenology in semi-arid and arid systems (Crimmins et al., 2011) while many prairie systems are driven - at varying times - by temperature and moisture (Craine et al., 2012b). In tropical regions, temperature, precipitation and irradiance may interact to produce the complexity of plant phenologies (van Schaik et al., 1993) - although teasing apart proximate cues from how phenologies are ultimately shaped by abiotic forces (e.g. for access to light or moisture) versus biotic forces is a major challenge.

Considering abiotic and biotic drivers separately may be inappropriate for many species; instead, a mixed model considering both drivers may provide a more complete picture. For example, in temperate systems, early-flowering species may be shaped most strongly by abiotic forces but exact timing within the early season is probably shaped additionally by selection to reduce competition for resources (Mosquin, 1971). This mixed-model perspective may explain why research on model organisms has found that complicated pathways and cascading cues, involving responses to temperature, photoperiod and sometimes precipitation, often underlie the timing of flowering (Wilczek et al., 2010). 

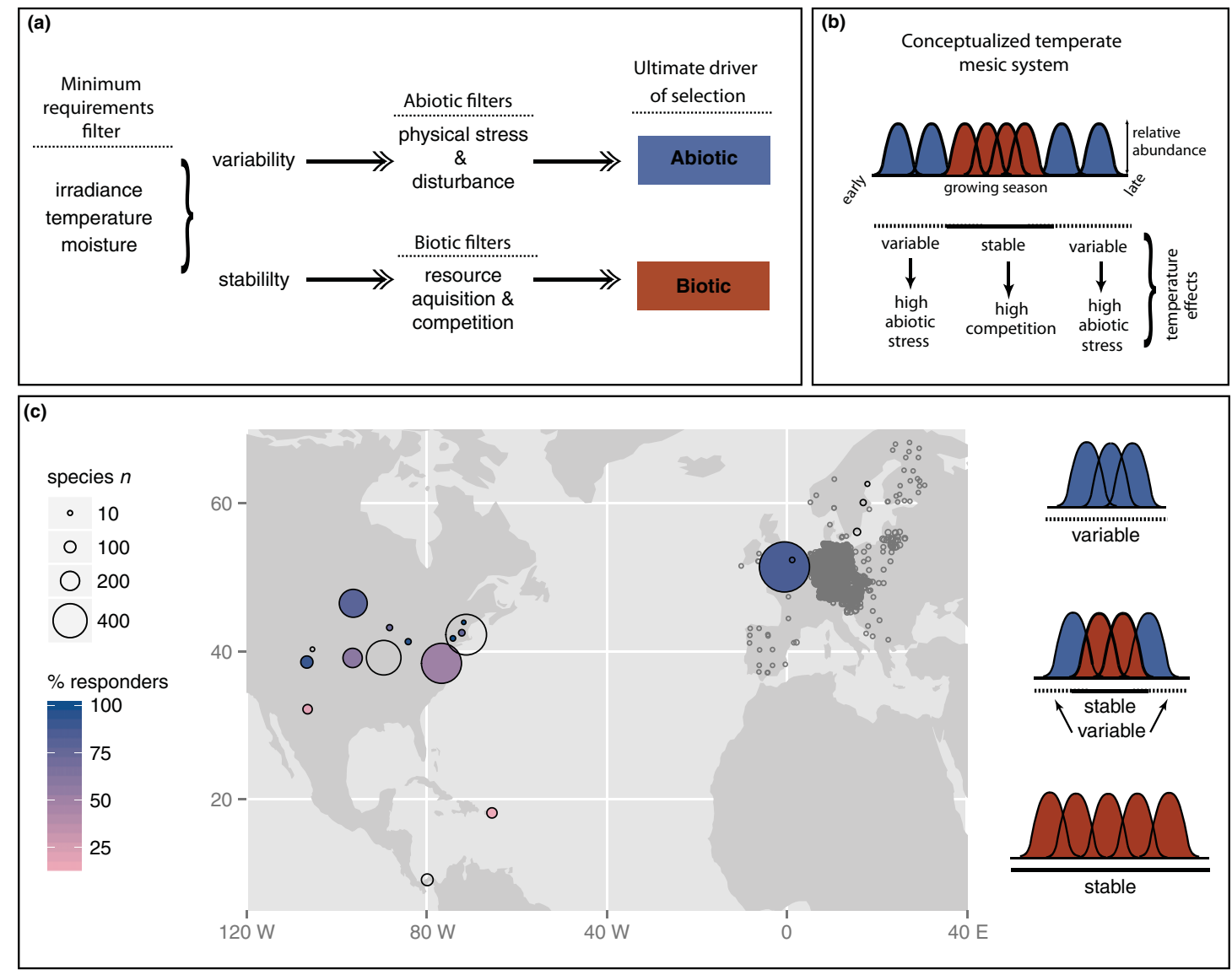

Box 1 Drivers of selection and climate variability across space and time

Coexistence theory suggests that species partition the growing season to minimize competition for resources (e.g. nutrients, light and pollinators; Gotelli \& Graves, 1996). Abiotic and biotic filters (a) should fundamentally shape such temporal niches (shown here as idealized distributions in $b-c$ ) through two dominant modes of evolution (a). Establishment requires that minimum resource requirements are met (minimum requirements filter in (a); we focus here on three dominant ones - temperature, moisture and irradiance - linked to plant phenology, which also control access to soil nutrients; van Schaik etal., 1993; Jolly et al., 2005). Once established, then variability versus stability in these three climatic factors should dictate the balance of biotic versus abiotic drivers of selection on shaping species' phenologies (a), and thus the balance between risk (e.g. early leafout leading to tissue loss caused by frost) and benefit (e.g. early access to soil nutrient resources).

Ultimate drivers of selection on phenology can then be either primarily abiotic (blue shading of niches) or biotic (red shading of niches). For species active during periods of high climatic variability, we expect that abiotic forces should dominantly control phenology, while phenology during periods of climatic stability may be governed more by biotic drivers, where competition between species for soil resources, light or pollinators strongly shapes selection (a). Because stability and variability vary across latitudes and within growing seasons, we thus predict that abiotic drivers might dominate in high latitudes and high-altitude communities with short, highly variable growing seasons, and at the start and end of mid-latitude temperate systems, while biotic factors might dominate to shape phenology during climatically stable mid-season periods, and in ever-wet tropical systems where variability in climate is low (b-c).

Currently, robust tests of these hypotheses are limited by the challenge in differentiating between underlying drivers and proximate cues, a geographical bias in existing data towards the temperate mid-latitudes (see map in c, which shows two major databases of plant phenology observations: sites with first flowering data from PEP725 (an extension of COST725; see Menzel etal. (2006)) are shown in gray circles, while flowering time data from NECTAR (http://knb.ecoinformatics.org/knb/metacat/nceas.988/knb) are shown in black circles) and rare availability of soil moisture and irradiance data. Thus, we show conceptualized examples focused on variability versus stability in temperature $(\mathrm{b}-\mathrm{c})$ and discuss additional drivers in the main text. In (c), shading in filled circles represents the percentage of species at a site that significantly respond to temperature, while unfilled circles represent sites without available time-series data. See Wolkovich etal. (2012) for more details on databases and site descriptions and Cook etal. (2012a) for details on temperature responses by sites. 

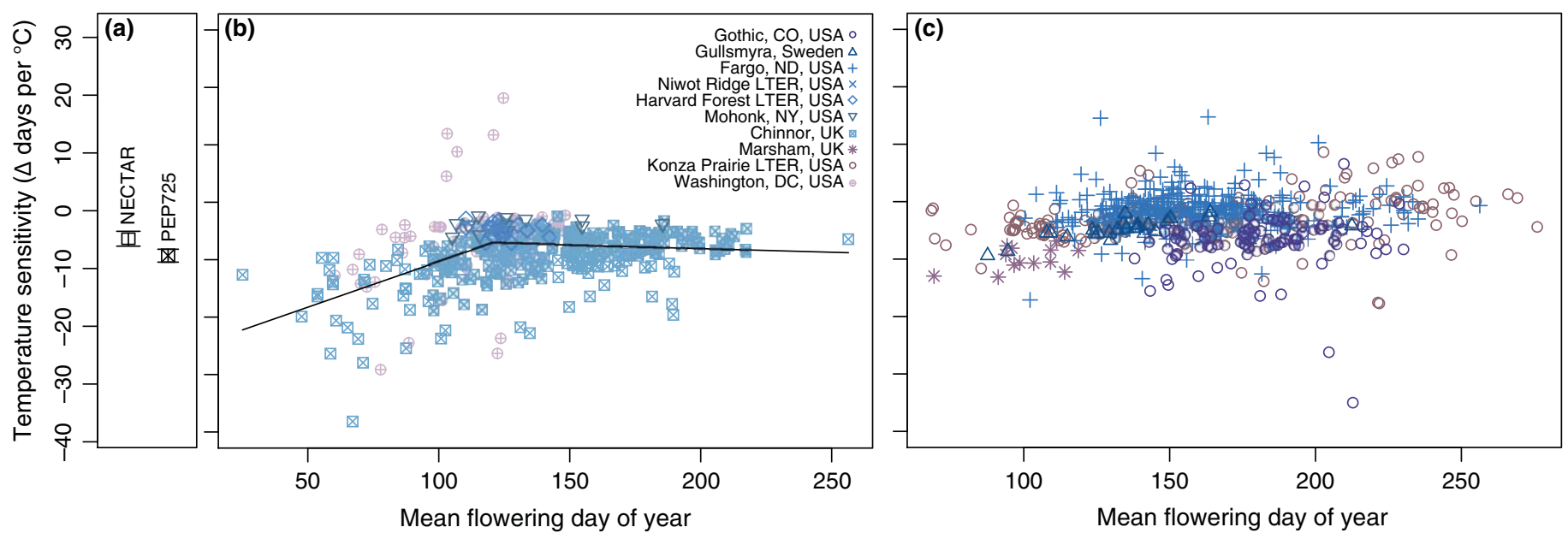

Fig 1 Mean temperature sensitivity of flowering (as measured by change in days per ${ }^{\circ} \mathrm{C}$ of mean annual temperature using first flowering date records) is highly consistent across diverse long-term records from Northern Hemisphere temperate sites (a, mean and standard errors shown; taken from Wolkovich et al., 2012). This mean, however, masks high variability between species (b-c), with some advancing their phenology up to a month per ${ }^{\circ} \mathrm{C}$ while others delay several weeks within the same location. Many studies using data focused on mesic temperate mid-latitude sites have now found that species that flower earlier in the season are most sensitive to temperature (Menzel et al., 2006; Cook et al., 2012a,b; Wolkovich et al., 2012). This higher sensitivity for early-flowering species usually breaks down well before the mid-season: we show (in b) a breakpoint model fit to the four sites occupying similar climate space to PEP725 (Chinnor, UK; Harvard Forest LTER; Mohonk, NY, USA and Washington, DC, USA), as significant breakpoint models for the sites outside this climate space (c) have not been found (Cook et al., 2012a). Sites are given in ascending order of their mean annual temperature; see Supporting Information Methods S1 for methodological details, and Wolkovich et al. (2012) for further details on sites and calculations of temperature sensitivity.

\section{Species diversity}

Testing for a shifting balance between abiotic and biotic drivers of phenology requires data covering a diversity of species from diverse climates. Until recently, estimates of plant phenological responses to climate have focused on either data sets from one location, often encompassing high local species diversity (Fitter \& Fitter, 2002), or fewer and sometimes clonally planted species at larger spatial scales (Schwartz et al., 2006). Even major efforts to aggregate European data, combining millions of phenological observations, still covered a relatively narrow climate space, centered on mesic, temperate systems, and included only several dozen species (Menzel et al., 2006; Cook et al., 2012a). Yet, increasing interest in the biological impacts of climate change have led to the availability of phenological data across a greater diversity of species and climates than ever before.

Recent efforts to build a species-rich and climatically broad database of plant phenology have provided some insights (Box 1). The NECTAR database - encompassing phenological data for over 5000 plant species with time-series data for over 1500 species - has reinforced previous estimates of a mean advance in flowering or leafing of 4-6d per ${ }^{\circ} \mathrm{C}$ (Wolkovich et al., 2012), surprisingly similar to previous estimates from species-poor databases (Fig. 1). Both of these estimates, however, are highly biased towards northern temperate latitudes (Box 1). Predictions for phenological responses to temperature based on European aggregated data quickly deteriorate for North American systems with major precipitation swings (e.g. grasslands and alpine meadow systems) and are even poorer for tropical and arid systems, showing that predictions only hold within the sampled climate space (Cook et al., 2012a). Nevertheless, these results - of declining predictability in species' phenologies with decreasing latitude (Box 1) - provide basic support for an increasing role of abiotic drivers in more variable climates. It should be noted, however, that these results are only for temperature (Cook etal., 2012a) and thus provide a very weak test: a better test would include estimates of species' responses to soil moisture and irradiance as well (see 'Critical data needs' section).

Mean estimates of phenological responses to temperature, in addition to being highly biased towards species from northern temperature latitudes, mask tremendous diversity in species' responses to temperature (Fig. 1). Thus, a major challenge is understanding and predicting such high variance between species. Following the model of a shifting balance (Box 1), it has now been shown that early-season species in temperate mesic systems appear to be most sensitive to temperature (Fig. 1); however, and in contrast to predictions, there is no symmetrical increase in temperature sensitivity in late-flowering species, as almost all species appear to flower well before the variability in autumnal temperatures begins. Additionally, such patterns generally break down outside of highly temperature-controlled systems (Jolly et al., 2005) as variability in other climatic factors becomes more critical to phenology (Cook et al., 2012a). Together, these findings suggest that the temporal niche within a growing season that each species occupies may help to predict species-specific sensitivities to climate, but more work is needed on teasing out the complexity of responses to temperature (Cook et al., 2012b) and other climatic factors (Jolly et al., 2005) before the development of useful models for most species will be possible.

Additionally, efforts to consider phenology as one functional trait within a plant's life history strategy (Craine et al., 2012a) have helped in understanding the diversity of phenological responses. Annual species are, on average, more sensitive to temperature than perennial species (Wolkovich etal., 2012), while within tree 
species, earlier ontogenetic stages (e.g. seedlings) appear to be more responsive to temperature than adult stages (Vitasse, 2013). These findings suggest an important role for an investment/risk trade-off where individuals with lower investments appear more responsive to temperature compared with individuals with greater investments. Such responsiveness often corresponds to greater risks (see 'Integrating risk and investment' section), but also possibly greater benefits. Species that are highly responsive to temperature also tend to have increased abundance or fitness with warming (Cleland et al., 2012). Additionally, multiple studies now show that exotic species studied in their introduced ranges appear to be more sensitive to climate compared with the surrounding native community, suggesting that responsive phenologies may be important to invasion success (Wolkovich \& Cleland, 2011; Wolkovich et al., 2013).

\section{Phylogenetic, genetic and plastic variation}

One additional key to improved predictions of phenological responses to climate is an enhanced understanding of the proximate abiotic cues that underlie phenological events within a growing season. Phylogeny - the shared evolutionary history of species may provide one starting point, as previous (Kochmer \& Handel, 1986) and recent efforts (Davis et al., 2010; Davies et al., 2013) show that related species are more likely to flower at similar times, albeit there has been mixed support for phylogenetic structure in modeled estimates of species' responses to climate (Davis et al., 2010; Wolkovich et al., 2013). This means that species cannot be considered as statistically independent, and studies considering the timing of phenological events will need to consider their shared evolutionary history and genetic background.

Molecular ecology clearly shows a strong genetic basis for phenology (Howe etal., 2003; Wilczek et al., 2010), whether driven by cues that produce high phenotypic plasticity in phenology across a species' range with little genetic variation (e.g. temperature sums) or cues, such as photoperiod, that produce a relatively static timing across years within sites. The plastic and genetic nature of phenological variation highlights a major hurdle, as it means that phenological data taken from field observations of wild plants include mixed signals. For example, observations from one location of a wide-ranging species that triggers flowering predominantly by temperature cues may not accurately capture the within-species variation - because the exact day will vary across sites with varying climates. Such geographical variation in phenotypes is probably common among plant traits. For phenological data, one possible solution is to use time-series data that allow modeling of plastic responses to interannual climate variation. This method, however, has its own limitations, as it assumes that cues are not locally adapted across species' ranges and may introduce bias based on the local climate sampled.

\section{Towards climate change predictions}

The research outlined above has clear extensions towards predicting how species have responded and will respond to climate change across time and space. A more robust framework, however, will need to address major research gaps, which we outline in the next two subsections.

\section{Critical data needs}

Building to robust predictions with climate change will require an investment in new data and methods. In particular, there is a strong need for observational field data outside the temperate mid-latitudes (Box 1) and a need for measurement of climatic drivers beyond temperature. Data on soil moisture, humidity, and irradiance (diffuse and direct) are necessary for any basic estimates of how phenological cues may vary across species, habitats and latitudes. However, climatic factors are generally intrinsically linked (e.g. cloud cover, radiation and precipitation often covary), making it difficult to separate effects fully. Thus, field, glasshouse and growth chamber experiments will continually be needed to test hypothesized cues and separate multiple climatic factors.

Controlled studies on cues - if conducted across populations may also help to estimate the prevalence by which intraspecific variation in phenology is controlled by phenotypic plasticity versus genotypic adaptation. Here, phenological data from arboreta and clonal plantings across latitudes may provide some insight, but welldesigned common garden studies for a much greater diversity of species will be essential. This is an important gap for improving predictions across species' ranges, and also for robustly incorporating phylogenetic approaches further. Phylogenetic comparative methods will also need to be developed - while currently they are well developed for analyzing species values, the integration of population data with macroevolutionary time-scales remains a challenge.

Robust predictions of how species will shift their phenologies with climate change will also require far better estimates of how rapidly phenological responses can evolve. While highly phenotypically plastic responses to climate may allow species to track climate change, recent research shows that local adaptation can be quite rapid. Common garden studies of exotic species introduced to Europe and North America several hundred years ago have documented variation of over a month in flowering time across populations taken from latitudinal transects spanning 1200$1800 \mathrm{~km}$ (Weber \& Schmid, 1998; Colautti et al., 2010). However, there may be a trade-off between plasticity and adaptation: phenotypic plasticity might allow species to match closely to the environment, but at the same time inhibit adaptive change by sheltering the species from strong selection (Price et al., 2003). Predictive models of plant phenology, other than those focused on very short time-scales relative to a species' generation time, will need to include estimates of evolutionary rates and the strength of selection coefficients (Anderson et al., 2012).

\section{Integrating risk and investment}

While variability in climatic factors may help to identify and predict species' phenological responses (Box 1), selection on plant phenology should be fundamentally driven by how such variability links to risk: species that leaf out too early in a temperate system risk tissue loss to frost (Inouye, 2008), while 
zoophilous species that flower before or after the main activity period of their pollinators may gain only a small payoff from their investment in flowers and nectar (Mosquin, 1971). Yet, highly risky phenological cues - for example, cuing strongly to early spring temperatures in temperate systems - may have big payoffs by allowing individuals early and longer access to resources. A critical area, thus, to improve predictions of plant phenological responses is a better model of how species and individuals balance risk and investment.

Investments - including in annual and woody growth and reproductive tissues - vary within and between species. For example, annual species with short-longevity, small seeds may have a lower investment than perennial species. Additionally, within species and within individuals, investment will vary across ontogeny and seasons: tree seedlings have lower investments than adult trees (Vitasse, 2013), while adult trees with pre-formed buds have a high investment. Given a similar risk environment, the investment model predicts that phenological cues that produce conservative responses to climate should scale positively with investment. This has generally been supported by research in temperate systems where annuals are more temperature-sensitive than perennial species (Wolkovich et al., 2012), and tree seedlings more temperature-sensitive than adults (Vitasse, 2013), while many adult trees with pre-formed buds have more complex and climate-conservative cues for breaking dormancy and leafing out in the spring (Howe et al., 2003). Building on these examples, however, requires more studies and quantitative estimates of investment - and risk - that are comparable across species, growing seasons and ecosystems.

Climate change introduces an important nonstationarity in risk - layering long-term directional shifts onto a system's annual and interannual climate variability. In many systems it appears that this nonstationarity favors species with highly plastic phenologies that allow them to track this new variability closely (Cleland et al., 2012). In other systems, however, shifts in extreme events with climate change appear to make plastic strategies riskier (Inouye, 2008). Thus, one important avenue to predicting system-specific optimum strategies will be to examine (1) how extreme events, relative to climatic means, have shifted and will shift with climate change and (2) how plants respond to these extreme events. To date there is little research in either of these areas (Reyer et al., 2013), and shifts in even one risk factor (e.g. frost) appear to vary across space (see Supporting Information Notes S1).

\section{Conclusions}

The increasing availability of phenological data alongside research in climate change has allowed researchers to make progress on fundamental questions of the scale and drivers of phenological variation across time, space and species. Recent efforts to integrate across climate science, ecology and evolution have highlighted consistent mean estimates of plant responses to temperature, but also major phenological variation between species and outside of the temperate mid-latitudes. Predicting future phenological responses to climate change will require an investment in new data, alongside the development of new methods and concepts that cut across disciplines. It will require molecular studies that extrapolate beyond model organisms to other species and clades, biometeorological studies that integrate genetic variation, and ecological studies across a phylogenetic diversity of species that scale up from local, short-term dynamics.

\section{Acknowledgements}

We thank D. Ackerly, M. O'Connor and three anonymous reviewers for help in improving this manuscript.

\section{References}

Anderson JT, Inouye DW, McKinney AM, Colautti RI, Mitchell-Olds T. 2012. Phenotypic plasticity and adaptive evolution contribute to advancing flowering phenology in response to climate change. Proceedings of the Royal Society B: Biological Sciences 279: 3843-3852.

Cleland EE, Allen JM, Crimmins TM, Dunne JA, Pau S, Travers SE, Zavaleta ES, Wolkovich EM. 2012. Phenological tracking enables positive species responses to climate change. Ecology 93: 1765-1771.

Cleland EE, Chuine I, Menzel A, Mooney HA, Schwartz MD. 2007. Shifting plant phenology in response to global change. Trends in Ecology \& Evolution 22: 357-365.

Colautti RI, Eckert CG, Barrett SCH. 2010. Evolutionary constraints on adaptive evolution during range expansion in an invasive plant. Proceedings of the Royal Society B: Biological Sciences 277: 1799-1806.

Cook BI, Wolkovich EM, Davies TJ, Ault TR, Betancourt JL, Allen JM, Bolmgren K, Cleland EE, Crimmins TM, Kraft NJB et al. 2012a. Sensitivity of spring phenology to warming across temporal and spatial climate gradients in two independent databases. Ecosystems 15: 1283-1294.

Cook BI, Wolkovich EM, Parmesan C. 2012b. Divergent responses to spring and winter warming drive community level flowering trends. Proceedings of the National Academy of Sciences, USA 109: 9000-9005.

Craine JM, Wolkovich EM, Towne EG. 2012a. The roles of shifting and filtering in generating community-level flowering phenology. Ecography 35: 1033-1038.

Craine JM, Wolkovich EM, Towne EG, Kembel SW. 2012b. Flowering phenology as a functional trait in a tallgrass prairie. New Phytologist 193: 673-682.

Crimmins TM, Crimmins MA, Bertelsen CD. 2011. Onset of summer flowering in a 'Sky Island' is driven by monsoon moisture. New Phytologist 191: 468-479.

Davies TJ, Wolkovich EM, Kraft NJB, Salamin N, Allen JM, Ault TR, Betancourt JL, Bolmgren K, Cleland EE, Cook BI et al. 2013. Phylogenetic conservatism in plant phenology. Journal of Ecology 101: 1520-1530.

Davis CC, Willis CG, Primack RB, Miller-Rushing AJ. 2010. The importance of phylogeny to the study of phenological response to global climate change. Philosophical Transactions of the Royal Society B: Biological Sciences365:3201-3213.

Fitter AH, Fitter RSR. 2002. Rapid changes in flowering time in British plants. Science 296: 1689-1691.

Gotelli NJ, Graves GR. 1996. The temporal niche. In: Gotelli NJ, Graves GR, eds. Null models in ecology. Washington, DC, USA: Smithsonian Institution, 95-111.

Howe GT, Aitken SN, Neale DB, Jermstad KD, Wheeler NC, Chen THH. 2003. From genotype to phenotype: unraveling the complexities of cold adaptation in forest trees. Canadian Journal of Botany 81: 1247-1266.

Inouye DW. 2008. Effects of climate change on phenology, frost damage, and floral abundance of montane wildflowers. Ecology 89: 353-362.

Jolly WM, Nemani R, Running SW. 2005. A generalized, bioclimatic index to predict foliar phenology in response to climate. Global Change Biology 11: 619-632.

Kochmer JP, Handel SN. 1986. Contraints and competition in the evolution of flowering phenology. Ecological Monographs 56: 303-325.

Menzel A, Sparks TH, Estrella N, Koch E, Aasa A, Ahas R, Alm-Kubler K, Bissolli P, Braslavska O, Briede A et al. 2006. European phenological response to climate change matches the warming pattern. Global Change Biology 12: 1969-1976. 
Mosquin T. 1971. Competition for pollinators as a stimulus for evolution of flowering time. Oikos 22: 398-402.

Pau S, Wolkovich EM, Cook BI, Davies TJ, Kraft NJB, Bolmgren K, Betancourt JL, Cleland EE. 2011. Predicting phenology by integrating ecology, evolution and climate science. Global Change Biology 17: 3633-3643.

Price T, Qvarnstrom A, Irwin D. 2003. The role of phenotypic plasticity in driving genetic evolution. Proceedings of the Royal Society B: Biological Sciences 270: 14331440.

Reyer CP, Leuzinger S, Rammig A, Wolf A, Bartholomeus RP, Bonfante A, de Lorenzi F, Dury M, Gloning P, Abou Jaoudé R et al. 2013. A plant's perspective of extremes: terrestrial plant responses to changing climatic variability. Global Change Biology 19: 75-89.

van Schaik CP, Terborgh JW, Wright SJ. 1993. The phenology of tropical forests adaptive significance and consequences for primary consumers. Annual Review of Ecology and Systematics 24: 353-377.

Schwartz MD, Ahas R, Aasa A. 2006. Onset of spring starting earlier across the Northern Hemisphere. Global Change Biology 12: 343-351.

Vitasse Y. 2013. Ontogenic changes rather than difference in temperature cause understory trees to leaf out earlier. New Phytologist 198: 149-155.

Weber E, Schmid B. 1998. Latitudinal population differentiation in two species of Solidago (Asteraceae) introduced into Europe. American Journal of Botany 85: 1110-1121.

Wilczek AM, Burghardt LT, Cobb AR, Cooper MD, Welch SM, Schmitt J. 2010. Genetic and physiological bases for phenological responses to current and predicted climates. Philosophical Transactions of the Royal Society B: Biological Sciences 365: 3129-3147.

Wolkovich EM, Cleland EE. 2011. The phenology of plant invasions: a community ecology perspective. Frontiers in Ecology and the Environment 9: 287-294.
Wolkovich EM, Cook BI, Allen JM, Crimmins TM, Betancourt JL, TraversSE, Pau S, Regetz J, Davies TJ, Kraft NJB et al. 2012. Warming experiments underpredict plant phenological responses to climate change. Nature 485: 494-497.

Wolkovich EM, Davies TJ, Schaefer H, Cleland EE, Cook BI, Travers SE, Willis CG, Davis C. 2013. Temperature-dependent shifts in phenology contribute to the success of exotic species with climate change. American Journal of Botany 100 $1407-1421$.

\section{Supporting Information}

Additional supporting information may be found in the online version of this article.

Table S1 Supporting information for Table 1

Methods S1 Methods for Fig. 1.

Notes S1 Extended discussion of shifting extreme events and phenology.

Please note: Wiley Blackwell are not responsible for the content or functionality of any supporting information supplied by the authors. Any queries (other than missing material) should be directed to the New Phytologist Central Office.

\section{About New Phytologist}

- New Phytologist is an electronic (online-only) journal owned by the New Phytologist Trust, a not-for-profit organization dedicated to the promotion of plant science, facilitating projects from symposia to free access for our Tansley reviews.

- Regular papers, Letters, Research reviews, Rapid reports and both Modelling/Theory and Methods papers are encouraged. We are committed to rapid processing, from online submission through to publication 'as ready' via Early View - our average time to decision is $<25$ days. There are no page or colour charges and a PDF version will be provided for each article.

- The journal is available online at Wiley Online Library. Visit www.newphytologist.com to search the articles and register for table of contents email alerts.

- If you have any questions, do get in touch with Central Office (np-centraloffice@lancaster.ac.uk) or, if it is more convenient, our USA Office (np-usaoffice@ornl.gov)

- For submission instructions, subscription and all the latest information visit www.newphytologist.com 